\title{
Assessing the $v^{2}-f$ Turbulence Models for Circulation Control Applications
}

\author{
Travis M. Storm*, David D. Marshall ${ }^{\dagger}$ \\ California Polytechnic State University, San Luis Obispo, California, 93407
}

\begin{abstract}
This paper explores the applicability of several variations of the $v^{2}-f$ turbulence model to circulation control flows. The effects of modifying the model to capture nonlinear eddy viscosity effects and streamline curvature effects are assessed. Results indicate that the $v^{2}-f$ turbulence model is capable producing more physically accurate results for circulation control flow fields than common modern turbulence modeling techniques.
\end{abstract}

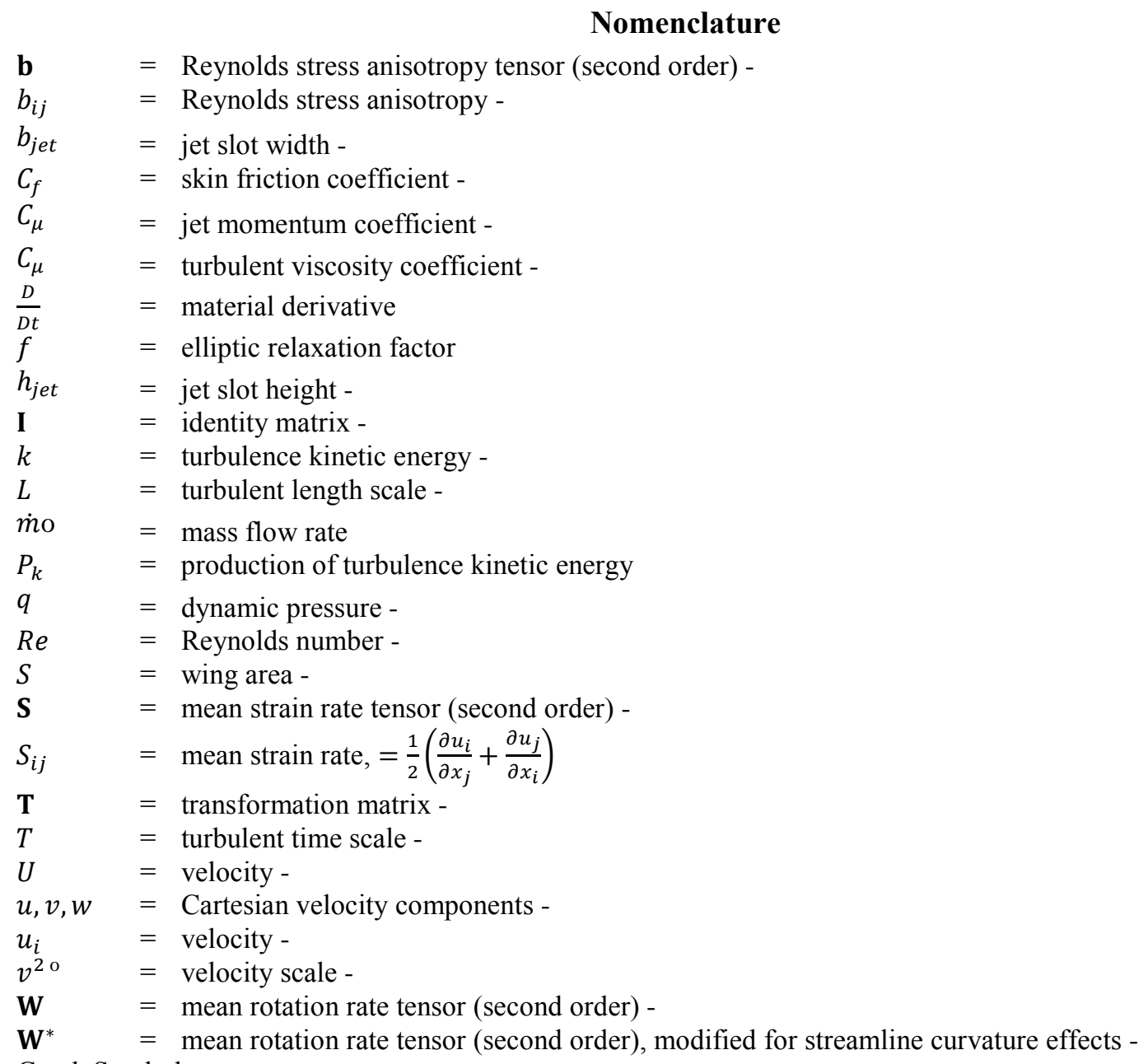

Greek Symbols

$\delta_{i j} \quad=$ Kronicker delta, $= \begin{cases}1, & i=j \\ 0, & i \neq j\end{cases}$

$\varepsilon \quad=$ turbulence dissipation rate -

\footnotetext{
${ }^{*}$ Student, Aerospace Engineering Dept., Student Member.

${ }^{\dagger}$ Associate Professor, Aerospace Engineering Dept., Senior Member. 


$\begin{array}{ll}\eta_{1} & =\text { strain invariant } \\ \eta_{2} & =\text { vorticity invariant } \\ \mu & =\text { laminar dynamic viscosity } \\ \mu_{t} & =\text { turbulent dynamic viscosity } \\ v & =\text { laminar kinematic viscosity } \\ v_{t} & =\text { turbulent kinematic viscosity } \\ \rho & =\text { density } \\ \tau & =\text { turbulent time scale } \\ \omega & =\text { specific dissipation rate } \\ \Omega_{i j} & =\text { mean rotation rate, }=\frac{1}{2}\left(\frac{\partial u_{i}}{\partial x_{j}}-\frac{\partial u_{j}}{\partial x_{i}}\right) \\ \bar{\Omega} & =\text { objective vorticity tensor }(\operatorname{second} \text { order })\end{array}$

Superscripts and Subscripts

$\infty \quad=$ freestream

\section{Introduction}

$\mathrm{T}$ O generate increased lift from traditional subsonic airfoils, either the angle of attack or the camber must be increased. The maximum lift coefficient of a traditional wing is limited by the eventual separation of flow over the wing, due primarily to the adverse pressure gradient that builds on the wing as lift is increased. Traditionally, this obstacle is overcome by use of complex moving wing surfaces, including flaps, slats, and other devices.

Circulation control has been proposed as a simpler and more effective alternative to the usual high-lift devices ${ }^{1}$. Circulation control is an active flow control device that increases the lift coefficient without the use of complex components in freestream flow. Circulation control is primarily needed when high lift coefficients are required due to low airspeeds, particularly during takeoff and landing. The technology makes use of the Coanda effect, according to which a fluid has a tendency to stay attached to an adjacent curved surface ${ }^{2}$. A high-speed jet of air is blown out of the leading and/or trailing edge of a wing, which follows the wing surface. The stagnation point on the leading edge and the flow separation point on the trailing edge are thus manipulated such that the circulation is increased, and consequently lift is increased.

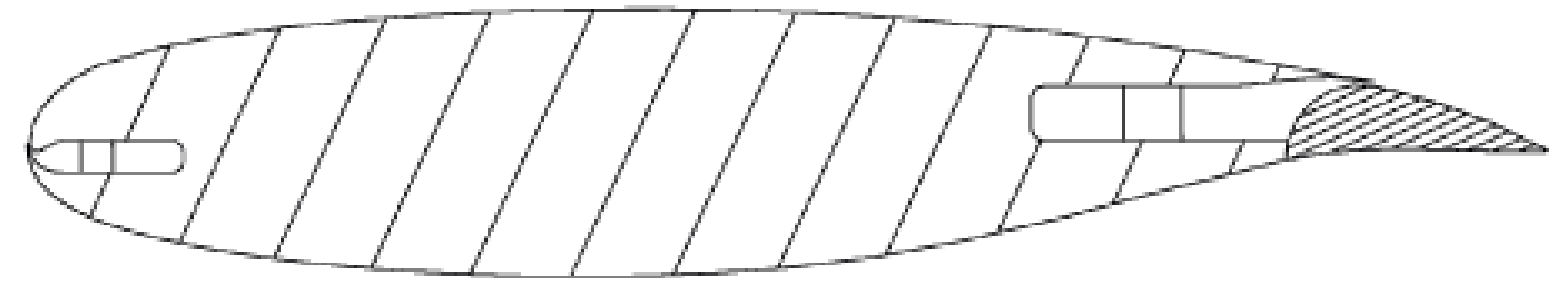

Figure 1. Circulation control airfoil with leading and trailing edge jets ${ }^{3}$.

The extent of the stagnation and separation point movement is primarily a function of the jet momentum coefficient, $C_{\mu}$. The jet momentum coefficient is a measure of the jet momentum relative to the freestream momentum, and has two common formulations, which are defined as follows.

$$
C_{\mu}=\frac{2 h_{j e t} b_{j e t}}{S} \frac{\rho_{j e t}}{\rho_{\infty}} \frac{U_{j e t}^{2}}{U_{\infty}^{2}}=\frac{\dot{m} U_{j e t}}{{ }_{\infty} S}
$$

The benefit of circulation control is that the lift is significantly increased, with relatively insignificant increases in drag. Circulation control requires high energy air to be blown over the wing's surface. This requires some additional source of energy, potentially the engine(s) or auxiliary gas generators; this is, of course, a problem associated with circulation control, but the solution is outside the scope of this research paper.

\section{Challenges to CFD Modeling of Circulation Control Flows}

\section{A. Streamline Curvature}

In the formulation of an algebraic stress model, the classical approach is to apply the weak-equilibrium assumption. When this assumption is applied to turbulence equations, the Reynolds stress anisotropy tensor $b_{i j}=$ 
$\frac{\overline{u_{i} u_{j}}}{-}-\frac{2}{3} \delta_{i j}$ is assumed to be constant along a streamline ${ }^{4}$. This assumption is untrue in flows with streamline curvature, and the effect is amplified in cases where such curvature is significant.

The calculation of material derivatives is necessary to solve the RANS equations. Material derivatives of scalar fields are Galilean invariant (i.e., invariant of the coordinate system;) however, material derivatives of tensor fields with rank greater than zero are not Galilean invariant ${ }^{4}$. The material derivative of the Reynolds stress anisotropy tensor is needed to solve the RANS equations, presenting a problem in flows with streamline curvature. Spalart and Shur ${ }^{5}$ proposed a method based on a Galilean invariant measure of turbulence for sensitizing eddy viscosity turbulence models to the effects of streamline curvature. Gatski et al. ${ }^{6}$ and Hellsten et al. ${ }^{7}$ proposed extensions of this approach, in which the anisotropy tensor is transformed to a local streamline-oriented coordinate system such that the weak-equilibrium assumption is valid. The general model for the transport of the anisotropy tensor is given as:

$$
\frac{D \mathbf{b}}{D t}=-\frac{\mathbf{b}}{a_{4}}-a_{3}\left(\mathbf{b S}+\mathbf{S b}-\frac{2}{3}\{\mathbf{b S}\} \mathbf{I}\right)+a_{2}(\mathbf{b} \mathbf{W}-\mathbf{W b})+\frac{a_{5} \varepsilon}{k}\left(\mathbf{b}^{2}-\frac{1}{3}\left\{\mathbf{b}^{2}\right\} \mathbf{I}\right)
$$

where $a_{i}$ are calibrated constants and \{\} represents the trace of a matrix. The weak-equilibrium assumption of this form gives $\frac{D \mathbf{b}}{D t}=0$, and leads to an algebraic system of equations for the Reynolds stress anisotropy tensor that is dependent on the choice of coordinate system. This can be corrected by introducing the transformation matrix $\mathbf{T}$, which accounts for the transformation from a global, inertial coordinate system to a local, streamline-oriented coordinate system. Hellsten et al. showed that taking the material derivative of $\mathbf{T b T}^{\mathrm{T}}$ and transforming back into the inertial coordinate system results in:

$$
\frac{D \mathbf{b}}{D t}=\mathbf{T}^{T}\left[\frac{D}{D t}\left(\mathbf{T} \mathbf{b} \mathbf{T}^{T}\right)\right] \mathbf{T}-(\mathbf{b} \overline{\mathbf{\Omega}}-\overline{\mathbf{\Omega}} \mathbf{b})
$$

where $\overline{\mathbf{\Omega}}=\mathbf{T} \frac{D \mathbf{T}^{T}}{D t}$. The transformation matrix must be selected such that the Reynolds stress anisotropy can be ignored in the local, streamline-oriented coordinate system. If this is true, the Reynolds stress anisotropy tensor transport equation simplifies to the following:

$$
\frac{D \mathbf{b}}{D t}=-(\mathbf{b} \overline{\mathbf{\Omega}}-\overline{\mathbf{\Omega}} \mathbf{b})
$$

This result can be incorporated into the original transport equation for the Reynolds stress anisotropy tensor by replacing $\mathbf{W}$ in Equation (2) with $\mathbf{W}^{*}=\frac{1}{a_{2}} \overline{\mathbf{\Omega}}$, resulting in the following, final form of the transport equation:

$$
\begin{aligned}
\frac{D \mathbf{b}}{D t}=-\frac{\mathbf{b}}{a_{4}}-a_{3} & \left(\mathbf{b S}+\mathbf{S b}-\frac{2}{3}\{\mathbf{b} \mathbf{S}\} \mathbf{I}\right)+a_{2}\left[\mathbf{b}\left(\mathbf{W}+\frac{1}{a_{2}} \overline{\mathbf{\Omega}}\right)-\left(\mathbf{W}+\frac{1}{a_{2}} \overline{\mathbf{\Omega}}\right) \mathbf{b}\right] \\
& +\frac{a_{5} \varepsilon}{k}\left(\mathbf{b}^{2}-\frac{1}{3}\left\{\mathbf{b}^{2}\right\} \mathbf{I}\right)
\end{aligned}
$$
T.

The problem of incorporating streamline curvature effects is then reduced to finding the transformation matrix,

\section{B. Eddy Viscosity Formulation}

The traditional approach to relating the turbulent Reynolds stresses to the mean strain rate tensor has been to make use of the Boussinesq assumption, thereby creating a linear eddy viscosity model (LEVM.) According to the Boussinesq assumption, the unnormalized Reynolds stresses and the mean strain rate tensor are related via the turbulent viscosity such that ${ }^{8}$

$$
\overline{u_{i} u_{j}}=\frac{2}{3} k \delta_{i j}-2 v_{t} S_{i j}
$$

Common LEVM formulations vary in complexity from zero-equation (algebraic) to four-equation; the number of equations refers to the number of differential equations that need to be solved in a given model. Examples of zeroequation LEVMs include the Cebeci-Smith ${ }^{9}$ and Baldwin-Lomax ${ }^{10}$ models. The most common one-equation LEVM is the Spalart-Allmaras turbulence model ${ }^{11}$. Two-equation LEVMs include the $k-\varepsilon^{12}$ and $k$ - $\omega^{13}$ turbulence models. The standard $v^{2}-f$ turbulence model ${ }^{14}$ is an increasingly common four-equation turbulence model. 
LEVMs have proven to be quite powerful in industrial and academic CFD applications, but the linearization of the relationship between the Reynolds stresses and the strain rate can cause these models to produce non-physical results. In particular, the Boussinesq assumption assumes that the eddy viscosity is isotropic; that is,

$$
\bar{u}^{2}=\bar{v}^{2}=\bar{w}^{2}=\frac{2}{3} k
$$

This negates any anisotropy of the Reynolds stresses, most notably near-wall anisotropy, which can be a significant feature of wall-bounded flows.

One approach to solving this shortcoming has been to introduce empirical damping functions or other sorts of ad-hoc modifications. This allows improvement upon a model for a given type of flow, but is far from universal. Pope $^{15}$ suggested that the more robust approach to this problem is to reformulate the relationship between the Reynolds stresses and the strain rate in a nonlinear manner. The general approach to formulating a nonlinear eddy viscosity model (NLEVM) is to generalize the formulation of the unnormalized Reynolds stresses to the following form ${ }^{16}$ :

$$
\overline{u_{i} u_{j}}=\frac{2}{3} k \delta_{i j}+k \sum_{\lambda} g^{\lambda} T_{i j}^{\lambda}
$$

where $T_{i j}^{\lambda}$ are the tensor bases and $g^{\lambda}$ are the calibrated expansion coefficients. The specific approach to deriving the general form of the Reynolds stresses can vary depending on the number and form of the terms chosen to include in the tensor bases.

\section{Previous CFD Approaches to Circulation Control Flows}

Traditional CFD approaches have been applied to circulation control airfoils, mostly in two dimensions, with mixed success. Unfortunately, the physics of circulation control wings are highly complex, and are not well understood due to limited experimental studies. The high momentum of the circulation control jet allows the boundary layer to remain attached longer than usual, thereby moving the separation point. This movement of the separation point is the primary reason that lift is augmented, and CFD modeling techniques must be able to accurately model the separation point by properly predicting the spreading rate of the jet and the exchange of momentum between the jet and the surrounding fluid.

Today's computer resources limit most academic and industrial CFD to RANS solutions, especially for high Reynolds number flows. Many attempts have been made to model circulation control flow fields using common turbulence models (including Baldwin-Lomax ${ }^{17,18,19}$, Spalart-Allmaras ${ }^{18,19,20,21,22,23,24}, k-\varepsilon^{25}$, and $k-\omega^{22,24,25,26}$,) and while acceptable accuracy has been obtained in some cases, the general consensus has been that these models are not well suited for circulation control flow fields.

One reason that common turbulence models poorly model circulation control flows is their use of the Boussinesq assumption. The Boussinesq assumption simplifies the relationship between the turbulent Reynolds stresses and the mean strain rate tensor to a linear relationship. Also, most common models do not capture streamline curvature effects, which might become significant in circulation control flows. Addressing these two issues for a robust turbulence model has the potential to greatly improve the CFD predictions of circulation control flows.

\section{The $v^{2}-f$ Turbulence Models}

\section{A. Standard $v^{2}-f$ Turbulence Model}

Durbin $^{27}$ developed the $v^{2}-f$ turbulence model to be used in flows in which near-wall turbulence is of significant importance, specifically flows with separation, recirculation, or heat transfer ${ }^{28}$. The model solves four transport equations, those for turbulence kinetic energy, turbulence dissipation rate, velocity scale, and elliptic relaxation factor. The model is essentially an extension of the $k-\varepsilon$ turbulence model, with the computational advantage of using the eddy viscosity concept to close the transport equations (as opposed to full second moment closure,) but improves upon several known deficiencies of the $k$ - $\varepsilon$ model. Specifically, the $v^{2}-f$ model can be integrated to a solid wall, eliminating the need for damping functions or wall functions ${ }^{29}$. Also, the introduction of the velocity scale allows the model to correctly scale damping of turbulence transport near walls, which turbulence kinetic energy is theoretically incapable of ${ }^{30}$. In recent years, the $v^{2}-f$ turbulence model has proven robust and superior to other RANS methods, despite its linear eddy viscosity formulation and insensitivity to streamline curvature ${ }^{28}$.

The $v^{2}-f$ turbulence model uses the same transport equations for turbulence kinetic energy and turbulence dissipation rate as does the $k-\varepsilon$ turbulence model. In addition to these transport equations, this model solves the following transport equations for the velocity scale and the elliptic relaxation factor:

4 -

American Institute of Aeronautics and Astronautics 2010-1054 


$$
\begin{gathered}
\frac{\partial \overline{v^{2}}}{\partial t}+u_{j} \frac{\partial \overline{v^{2}}}{\partial x_{j}}=k f-\overline{v^{2}} \frac{\varepsilon}{k}+\frac{\partial}{\partial x_{j}}\left[\left(v+\frac{v_{t}}{\sigma}\right) \frac{\partial \overline{v^{2}}}{\partial x_{j}}\right] \\
L^{2} \frac{\partial f^{2}}{\partial x_{j}}-f=\frac{1}{T}\left(C_{1}-1\right)\left(\frac{\overline{v^{2}}}{k}-\frac{2}{3}\right)-C_{2} \bar{k}
\end{gathered}
$$

where represents the production of turbulence kinetic energy due to the mean flow velocity gradients, and is modeled as

$$
=2 \mu_{t} S^{2}
$$

The turbulence length scale, $L$, and the turbulence time scale, $T$, are defined as follows.

$$
\begin{gathered}
L=C_{L} \max \left[\frac{k^{3 / 2}}{\varepsilon}, C_{\eta}\left(\frac{\mu^{3}}{\rho^{3} \varepsilon}\right)^{1 / 4}\right] \\
T=\max \left[\frac{k}{\varepsilon}, C_{T} \sqrt{\frac{\mu}{\rho \varepsilon}}\right]
\end{gathered}
$$

The turbulent viscosity is defined as:

$$
\mu_{t}=\rho C_{\mu} \overline{v^{2}} T
$$

It is important to note that this model does not use any wall functions or damping functions. Instead, the model uses the velocity scale (which is a measure of velocity fluctuation normal to streamlines) to damp turbulence transport near inhomogeneities, and the elliptic relaxation factor to model non-local effects.

Kalitzin $^{31}$ applied the $v^{2}-f$ turbulence model to simple aerospace configurations, and compared the results to those produced by Menter's $k$ - $\omega$ turbulence model and the Spalart-Allmaras turbulence model. Results were generated for a subsonic A-airfoil, a transonic RAE2822 airfoil, and a subsonic three-element trapezoidal wingbody. Given that the configurations are simple airfoils and a subsonic wing-body, the cases are considered validation cases for the $v^{2}-f$ turbulence model. In all cases, the results between the three models matched quite well, indicating that the $v^{2}-f$ turbulence model correctly predicts flows for simple aerospace configurations.

$\mathrm{Bell}^{28}$ performed an in-depth comparison of turbulence models for a wide variety of flows; the turbulence models included in the comparison include, but are not limited to, Launder-Sharma, Spalart-Allmaras, standard $k-\varepsilon$, realizable $k-\varepsilon, k-\varepsilon-R N G$, standard $k-\omega$, Menter's $k-\omega$, and standard $v^{2}-f$. The test cases include a fully developed channel flow, an asymmetric planar diffuser, an axisymmetric afterbody, a low Reynolds number flow over a backstep, and an impinging jet. In every case in which the standard $v^{2}-f$ turbulence model was used, it produced results similar to or more accurate than those from other RANS models.

\section{B. Nonlinear $v^{2}-f$ Turbulence Model}

One deficiency in the standard $v^{2}-f$ turbulence model is the use of the Boussinesq assumption to linearize the relationship between the Reynolds stresses and the mean strain rate. Pettersson Reif ${ }^{32}$ proposed a nonlinear constitutive relationship that could account for turbulence anisotropy, thereby improving the $v^{2}-f$ model's predictive capability for turbulent shear flows. The nonlinearization begins with the proposal by Pope ${ }^{6}$ for an equilibrium solution of a second-moment closure for an incompressible, two-dimensional mean flow in a non-inertial frame,

$$
\frac{\overline{u u}}{k}=\frac{2}{3} \mathbf{I}-a_{1} \tau_{1} \mathbf{S}-a_{1} \tau^{2}\left(\mathbf{S W}^{*}-\mathbf{W}^{*} \mathbf{S}\right)+a_{3} \tau^{2}\left(\mathbf{S}^{2}-\frac{1}{3}\left|\mathbf{S}^{2}\right| \mathbf{I}\right)
$$

where the $a_{i}$ coefficients are functions of turbulent quantities, $\tau$ is a turbulent time scale, and $|\mathbf{S}|^{2}=S_{l} S_{l}$ (where $\mathbf{S}$ is a second order tensor.). Pettersson Reif used this form to propose the following relation for the $v^{2}-f$ turbulence model:

$$
\frac{\overline{u u}}{k}=\frac{2}{3} \mathbf{I}-C_{\mu 1}^{*} \frac{\bar{v}^{2}}{k} \tau_{1} \mathbf{S}-C_{\mu 2}^{*} V_{1} \tau^{2}\left(\mathbf{S W}^{*}-\mathbf{W}^{*} \mathbf{S}\right)+C_{\mu 3}^{*} V_{2} \tau^{2}\left(\mathbf{S}^{2}-\frac{1}{3}\left|\mathbf{S}^{2}\right| \mathbf{I}\right)
$$

where $V_{i}$ and $C_{\mu i}^{*}$ are functions of $\frac{\overline{v^{2}}}{-}$ and the dimensionless velocity-gradient parameters

$$
\begin{gathered}
\eta_{1}=\tau^{2} S_{i} S_{i} \\
\eta_{2}=\tau^{2} W_{i}^{*} W_{i}^{*}
\end{gathered}
$$

For two-dimensional incompressible flow, Equation (2) gives the following nonzero components of the Reynolds stress tensor.

$$
\frac{\overline{u_{1}^{2}}}{k}=\frac{2}{3}-2 C_{\mu 1}^{*} \frac{v^{2}}{k} \tau_{1} \lambda+\frac{1}{3} C_{\mu 3}^{*} V_{2} \tau^{2} \lambda^{2}
$$




$$
\begin{gathered}
\frac{\overline{u_{2}^{2}}}{k}=\frac{2}{3}+2 C_{\mu 1}^{*} \frac{v^{2}}{k} \tau_{1} \lambda+\frac{1}{3} C_{\mu 3}^{*} V_{2} \tau^{2} \lambda^{2} \\
\frac{\overline{u_{3}^{2}}}{\frac{k}{u_{1} u_{2}}}=\frac{2}{3}-\frac{1}{3} C_{\mu 3}^{*} V_{2} \tau^{2} \lambda^{2} \\
=-2 C_{\mu 2}^{*} V_{1} \tau^{2} \omega \lambda
\end{gathered}
$$

In the model proposed by Pettersson Reif, the principles of realizability and internal consistency were used to determine the final form of the nonlinear $v^{2}-f$ turbulence model. Realizability is the requirement that all quantities known to be strictly positive must be guaranteed to be positive by the turbulence model ${ }^{33}$. Specifically, for the Reynolds stresses, the following three relations constitute realizability.

$$
\begin{gathered}
\overline{u_{\propto}^{2}} \geq 0 \\
\overline{u_{\propto}^{2}} \leq k \\
\left(\overline{u_{\propto} u_{\beta}}\right)^{2} \leq u_{\propto}^{2} u_{\beta}^{2}
\end{gathered}
$$

It was assumed that the coefficient $C_{\mu 1}^{*}$ would take the same form as that proposed by Pettersson Reif et al. ${ }^{34}$ for the streamline curvature corrected $v^{2}$-f turbulence model. Applying the constraints in Equation (8) to Equations (4)(7) results in the following forms for the coefficients $C_{\mu 1}^{*}$ :

$$
\begin{gathered}
C_{\mu 1}=C_{\mu} F \\
C_{\mu 2}^{*}=\frac{\beta_{0} A}{\beta_{1}+V_{10} \sqrt{\eta_{1} \eta_{2}}} \\
C_{\mu 3}^{*}=\frac{\gamma_{0}}{\gamma_{1}+V_{20} \eta_{1}}
\end{gathered}
$$

where $\beta_{0}=\gamma_{0}=1$ and the coefficients $\beta_{1} \ll 1$ and $\gamma_{1} \ll 1$ exist only to prevent singularities.

The remaining unknowns can be determined by applying the concept of internal consistency, according to which the nonlinear constitutive relation should reduce to $\overline{u_{2}^{2}} \approx \overline{v^{2}}$ in parallel shear flow and an inertial reference frame. In such a flow, $S_{12}=S_{21}=W_{12}^{*}=-W_{21}^{*}$, and the diagonal elements of the Reynolds stress tensor can be written as:

$$
\begin{gathered}
\frac{\overline{u_{1}^{2}}}{\frac{k}{u_{2}^{2}}}=\frac{2}{k}+C_{\mu 2}^{*} V_{1} \eta_{1}+\frac{1}{6} C_{\mu 3}^{*} V_{2} \eta_{1} \\
=\frac{2}{\frac{3}{u_{3}^{2}}}-C_{\mu 2}^{*} V_{1} \eta_{1}+\frac{1}{6} C_{\mu 3}^{*} V_{2} \eta_{1} \\
\frac{2}{k}-\frac{1}{3} C_{\mu 3}^{*} V_{2} \eta_{1}
\end{gathered}
$$

Internal consistency requires that $\overline{u_{2}^{2}}$ reduces to $\overline{v^{2}}$ in parallel shear flow and an inertial reference frame; that is,

Given that

$$
\frac{\overline{u_{2}^{2}}}{k} \rightarrow \frac{\overline{v^{2}}}{k}=\frac{2}{3}-\beta_{0} A \frac{V_{1}}{V_{10}}+\frac{1}{6} \gamma_{0} \frac{V_{2}}{V_{20}}
$$

$$
\frac{V_{1}}{V_{10}}=\frac{V_{2}}{V_{20}}=\frac{6}{5}\left(\frac{2}{3}-\frac{\overline{v^{2}}}{k}\right)
$$

the nonlinear $v^{2}-f$ turbulence model is fully defined. The final model is described by the following equation set.

$$
\begin{aligned}
\overline{u_{i} u_{j}}=\frac{2}{3} k \delta_{i j}- & 2 C_{\mu 1}^{*} \overline{v^{2}} \tau_{1} S_{i j} \\
& -V \tau^{2}\left[C_{\mu 2}^{*}\left(S_{i} W^{*}{ }_{j}+S_{j} W_{i}^{*}\right)-C_{\mu 3}^{*}\left(S_{i} S_{j}-\frac{1}{3}\left|S^{2}\right| \delta_{i j}\right)\right]
\end{aligned}
$$

where

$$
\begin{gathered}
C_{\mu 1}=C_{\mu} F \\
C_{\mu 2}^{*}=\frac{6}{5} \frac{\sqrt{1-\left(C_{\mu 1}^{*} \frac{v^{2}}{k}\right)^{2} 2 \eta_{1}}}{\beta_{1}+\sqrt{\eta_{1} \eta_{2}}} \\
C_{\mu 3}^{*}=\frac{6}{5} \frac{1}{\gamma_{1}+\eta_{1}} \\
6-
\end{gathered}
$$

American Institute of Aeronautics and Astronautics 2010-1054 


$$
\begin{gathered}
V=\max \left(\frac{2}{3}-\frac{\overline{v^{2}}}{k}, 0\right) \\
\beta_{1}=\frac{1}{0.1+\overline{\eta_{1} \eta_{2}}} \\
\gamma_{1}=\frac{1}{0.1+\eta_{1}}
\end{gathered}
$$

Heschl et al. ${ }^{35}$ used the nonlinear formulation of the $v^{2}-f$ turbulence model to solve a flow in a room with threedimensional wall jets, and compared the results to their experimental data collected using PIV as well as results for many other turbulence models. The nonlinear $v^{2}-f$ turbulence model proved robust enough to capture secondary flows generated by the wall jets, a flow phenomenon that is attributed to Reynolds stress anisotropy and was not predicted by any other eddy viscosity turbulence models.

\section{Nonlinear $v^{2}-f$ Turbulence Model with Streamline Curvature Correction}

Another deficiency in the $v^{2}-f$ turbulence model is its insensitivity to streamline curvature. Two general approaches have been attempted to sensitize turbulence models to streamline curvature. First, the turbulent viscosity coefficient can be sensitized to invariants of strain and vorticity by modifying the $\eta_{1}$ and $\eta_{2}$ formulations. Or, the production and dissipation terms can be modified. Duraisamy et al. ${ }^{16}$ modified the $v^{2}-f$ turbulence model according to the first approach.

In the standard $v^{2}-f$ turbulence model, the anisotropy tensor is

$$
b_{i j}=C_{\mu} \frac{v^{2}}{k} \frac{k}{\varepsilon} S_{i j}
$$

This formulation is based on the assumption of Galilean invariance, according to which the anisotropy tensor should be the same in any coordinate system. Unfortunately, for flows with streamline curvature, this formulation of the anisotropy tensor is only valid in a streamline-oriented coordinate system. As a consequence of this, Duraisamy concludes that the anisotropy tensor is unable to capture streamline curvature effects, and these effects need to be incorporated explicitly. The formulation for streamline curvature effects begins with work by Pettersson Reif et al. ${ }^{34}$, in which the $v^{2}-f$ turbulence model was sensitized to frame-rotation effects (a non-inertial effect that also needed to be explicitly introduced.) This work consequently sensitized the vorticity invariant term to the mean vorticity tensor, which accounts for some streamline curvature effects. This work led to the following formulations for the strain and vorticity invariants:

$$
\begin{gathered}
\eta_{1}=\frac{k^{2}}{\varepsilon^{2}}\left|\left[\frac{1}{2}\left(\frac{\partial u_{i}}{\partial x_{j}}+\frac{\partial u_{j}}{\partial x_{i}}\right)\right]\right|^{2} \\
\eta_{2}=\frac{k^{2}}{\varepsilon^{2}}\left|\left[\frac{1}{2}\left(\frac{\partial u_{i}}{\partial x_{j}}-\frac{\partial u_{j}}{\partial x_{i}}\right)\right]+C_{\omega} \Omega_{i j}\right|^{2} \\
\eta_{3}=\eta_{1}-\eta_{2}
\end{gathered}
$$

where the $\Omega_{i j}$ term is the angular velocity of the reference frame and $|\mathbf{X}|^{2}=X_{l} X_{l}$ (where $\mathbf{X}$ is a second order tensor.) The angular velocity term is neglected in the formulation of the streamline curvature corrected $v^{2}-f$ turbulence model, as turbulence models applied to circulation control flows do not need to account for reference frame rotation.

The turbulent viscosity coefficient is sensitized as follows.

$$
C_{\mu}^{*}\left(\eta_{1}, \eta_{2}\right)=C_{\mu} \frac{1+\alpha_{2}\left|\eta_{3}\right|+\alpha_{3}\left|\eta_{3}\right|}{1+\alpha_{4}\left|\eta_{3}\right|}\left[\sqrt{\frac{1+\alpha_{5} \eta_{1}}{1+\alpha_{5} \eta_{2}}}+\alpha_{1} \overline{\eta_{2}} \overline{\left|\eta_{3}\right|-\eta_{3}}\right]^{-1}
$$

where the $\alpha_{i}$ coefficients are 


$$
\begin{gathered}
\alpha_{1}=0.055 \overline{f_{1}} \\
\alpha_{2}=\frac{1}{2} f_{1} \\
\alpha_{3}=\frac{1}{4} f_{1} \\
\alpha_{4}=\frac{1}{5} \overline{f_{1}} \\
\alpha_{5}=\frac{1}{40}
\end{gathered}
$$

and

$$
f_{1}=\sqrt{\frac{\left(\frac{\overline{v^{2}}}{k}\right)}{\left(\frac{\bar{v}^{2}}{k}\right)_{\infty}}}, \quad\left(\frac{\overline{v^{2}}}{}\right)_{\infty}=0.367
$$

Note that in homogeneous shear flow, $\left(\frac{\overline{v^{2}}}{-}\right)=\left(\frac{\overline{v^{2}}}{-}\right)_{\infty}$, so $f_{1}=1$ and there is no need for, or application of, curvature correction.

This formulation of the $v^{2}-f$ turbulence model, sensitized to frame-rotation effects, has shown some improvement for flows with streamline curvature. Duraisamy ${ }^{41}$ showed that, in a wingtip vortex, the standard $v^{2}-f$ turbulence model predicts high eddy viscosity at the center of the vortex; this is unphysical, as a wingtip vortex acts similar to a rotating solid body, which has a stabilizing effect near the center of rotation. Pettersson Reif's frame-rotation modification improves prediction by reducing the eddy viscosity near the center of the vortex, but still shows relatively high eddy viscosity near the exterior of the vortex. Consequently, Duraisamy et al. further modified the $v^{2}-$ $f$ turbulence model to explicitly incorporate a streamline curvature correction.

The explicit introduction of streamline curvature correction was approached by adding an antisymmetric objective vorticity tensor that results from a transformation from the global coordinate frame to a streamlineoriented coordinate frame. Methods for determining this tensor are generally classified into two categories, acceleration-based and strain-based, of which the latter has proven more robust ${ }^{36}$. For the $v^{2}-f$ turbulence model, the vorticity invariant is redefined to include this new term:

$$
\eta_{2}=\left(\frac{k}{\epsilon}\right)^{2} \frac{1}{2}\left(\frac{\partial u_{i}}{\partial x_{j}}-\frac{\partial u_{j}}{\partial x_{i}}\right)+C\left(\Omega_{i j}+\bar{\Omega}_{i j}\right)
$$

where, for non-rotating reference frames, $\Omega_{i j}=0$ and $\bar{\Omega}_{i j}=-\varepsilon_{i j} \bar{\omega}$ is the objective vorticity tensor. According to Wallin et al. ${ }^{4}$, the vorticity term is determined as:

$$
\bar{\omega}_{i}=\frac{\Pi_{1}^{2} \delta_{i j}+12 \Pi_{2} S_{i j}+6 \Pi_{1} S_{i} S_{j}}{2 \Pi_{1}^{3}-12 \Pi_{2}^{2}} S_{p l} S_{l q}^{\prime} \varepsilon_{p q j}
$$

where $\Pi_{1}=\operatorname{trace}\left(\mathbf{S}^{2}\right), \Pi_{2}=\operatorname{trace}\left(\mathbf{S}^{3}\right)$, and ()$^{\prime}$ is a material derivative. The Einstein summation notation of Equation (2) is convenient for its conciseness, but is not well-suited for programming. For the sakes of clarity and completeness, the expansion of the objective vorticity tensor $\bar{\Omega}_{i j}$ is shown as follows.

$$
\left[\begin{array}{lll}
\bar{\Omega}_{11} & \bar{\Omega}_{12} & \bar{\Omega}_{13} \\
\bar{\Omega}_{21} & \bar{\Omega}_{22} & \bar{\Omega}_{23} \\
\bar{\Omega}_{31} & \bar{\Omega}_{32} & \bar{\Omega}_{33}
\end{array}\right]=\left[\begin{array}{ccc}
0 & -\omega_{3} & \omega_{2} \\
\omega_{3} & 0 & -\omega_{1} \\
-\omega_{2} & \omega_{1} & 0
\end{array}\right]
$$

where

$$
\left[\begin{array}{l}
\omega_{1} \\
\omega_{2} \\
\omega_{3}
\end{array}\right]=\frac{\Pi_{1}^{2}\left[\begin{array}{lll}
1 & 0 & 0 \\
0 & 1 & 0 \\
0 & 0 & 1
\end{array}\right]+12 \Pi_{2}\left[\begin{array}{lll}
S_{11} & S_{12} & S_{13} \\
S_{21} & S_{22} & S_{23} \\
S_{31} & S_{32} & S_{33}
\end{array}\right]+6 \Pi_{1}\left[\begin{array}{lll}
S_{11} & S_{12} & S_{13} \\
S_{21} & S_{22} & S_{23} \\
S_{31} & S_{32} & S_{33}
\end{array}\right]}{2 \Pi_{1}^{3}-12 \Pi_{2}^{2}}\left[\begin{array}{l}
S_{23} S_{23}^{\prime}-S_{32} S_{32}^{\prime} \\
S_{31} S_{31}^{\prime}-S_{13} S_{13}^{\prime} \\
S_{12} S_{12}^{\prime}-S_{21} S_{21}^{\prime}
\end{array}\right]
$$

Duraisamy et al. applied the curvature corrected $v^{2}-f$ turbulence model to a NACA 0012 airfoil, and results were compared to the standard $v^{2}-f$ turbulence model, the $v^{2}-f$ model with frame-rotation effects, and experimental data collected by Chow et al. ${ }^{37}$ These results indicate considerable improvement for wingtip vortex flows, both in prediction of the mean velocity field and turbulence kinetic energy, though Duraisamy et al. warn that the results should be considered preliminary because of the lack of other validation cases.

$8-$

American Institute of Aeronautics and Astronautics 2010-1054 


\section{Calibration of the Models}

The constants used in the $v^{2}-f$ turbulence model have been altered and calibrated numerous times since the model was first introduced. Many authors make logical arguments for the order of magnitude of the constants, but the constants have yet to be systematically calibrated (at least as far as the authors of this paper are aware.) To address this issue, the constants were calibrated for a turbulent flow over a flat plate. Particular attention was paid to two aspects of the flat plate flow. First, the skin friction profile needed to match the well-known experimental profile defined as follows

$$
C_{f}=\frac{0.455}{\ln ^{2}\left(0.06 R e_{x}\right)}
$$

Second, the model was tuned such that the near-wall velocity profile matched the widely known velocity profiles in the laminar sublayer, buffer region, and into the log region. The velocity profile in the laminar sublayer follows a linear relationship between the velocity and distance from the wall, the velocity profile in the buffer layer is defined by Spalding's Law of the Wall, and velocity profile in the logarithmic overlap region follows a logarithmic relationship ${ }^{43}$. These three relationships are, respectively,

$$
\begin{array}{cc}
u^{+}=y^{+} & \text {for } y^{+}<5 \\
u^{+}=-y^{+}+e^{\kappa B}\left[e^{\kappa u^{+}}-1-\kappa u^{+}-\frac{1}{2}\left(\kappa u^{+}\right)^{2}-\frac{1}{6}\left(\kappa u^{+}\right)^{3}\right] & \text { for } 5<y^{+}<30 \\
u^{+}=\frac{1}{\kappa} \ln \left(u^{+}\right)+B & \text { for } 30<y^{+}<350
\end{array}
$$

where $\kappa=0.41$ and $B=5.0$.

The constants that make the model most closely match these criteria are as follows, for the three $v^{2}-f$ turbulence models ${ }^{44}$.

Table 1. Calibrated Constants for $v^{2}-f$ Turbulence Models

\begin{tabular}{|l|c|c|c|c|c|c|c|c|c|}
\hline & $C_{\mu}$ & $C_{1}$ & $C_{2}$ & $C_{L}$ & $C_{\eta}$ & $C E_{D}$ & $C E_{2}$ & $\sigma$ & $\sigma_{\varepsilon}$ \\
\hline Linear $v^{2}-f$ & 0.200 & 1.6 & 0.3 & 0.23 & 60 & 0.05 & 1.9 & 1.0 & 1.3 \\
\hline Nonlinear $v^{2}-f$ & 0.205 & 1.6 & 0.3 & 0.23 & 60 & 0.05 & 1.9 & 1.0 & 1.3 \\
\hline Linear $v^{2}-f-c c$ & 0.205 & 1.6 & 0.3 & 0.23 & 60 & 0.05 & 1.9 & 1.0 & 1.3 \\
\hline
\end{tabular}

\section{E. Application of the $v^{2}-f$ Turbulence Models in FLUENT}

The three above-mentioned variations on the $v^{2}-f$ turbulence model were written as user-defined functions to be used in conjunction with the commercial CFD solver FLUENT ${ }^{38}$. The standard and nonlinear $v^{2}-f$ models were written by Heschl et al. ${ }^{35,39}$, and were graciously provided for this research. Several modifications have been made to the provided codes to improve stability, robustness, and accuracy. The nonlinear $v^{2}-f$ model with curvature correction was written by the authors of this paper.

\section{Preliminary Results}

\section{A. Validation Case: Flat Plate in Laminar, Subsonic Flow}

The three $v^{2}-f$ models and several common turbulence models were used to solve a subsonic flow over a flat plate. The Reynolds number at the end of the plate is $9 \times 10^{4}$, indicating laminar flow. The skin friction coefficient generated using the $v^{2}-f$ turbulence models, several common turbulence models, and a theoretical relationship are shown in the following figure. The theoretical relationship is the well-known relationship for laminar flat plate skin friction coefficient ${ }^{40}$,

$$
C_{f}=\frac{0.664}{\overline{R e_{x}}}
$$




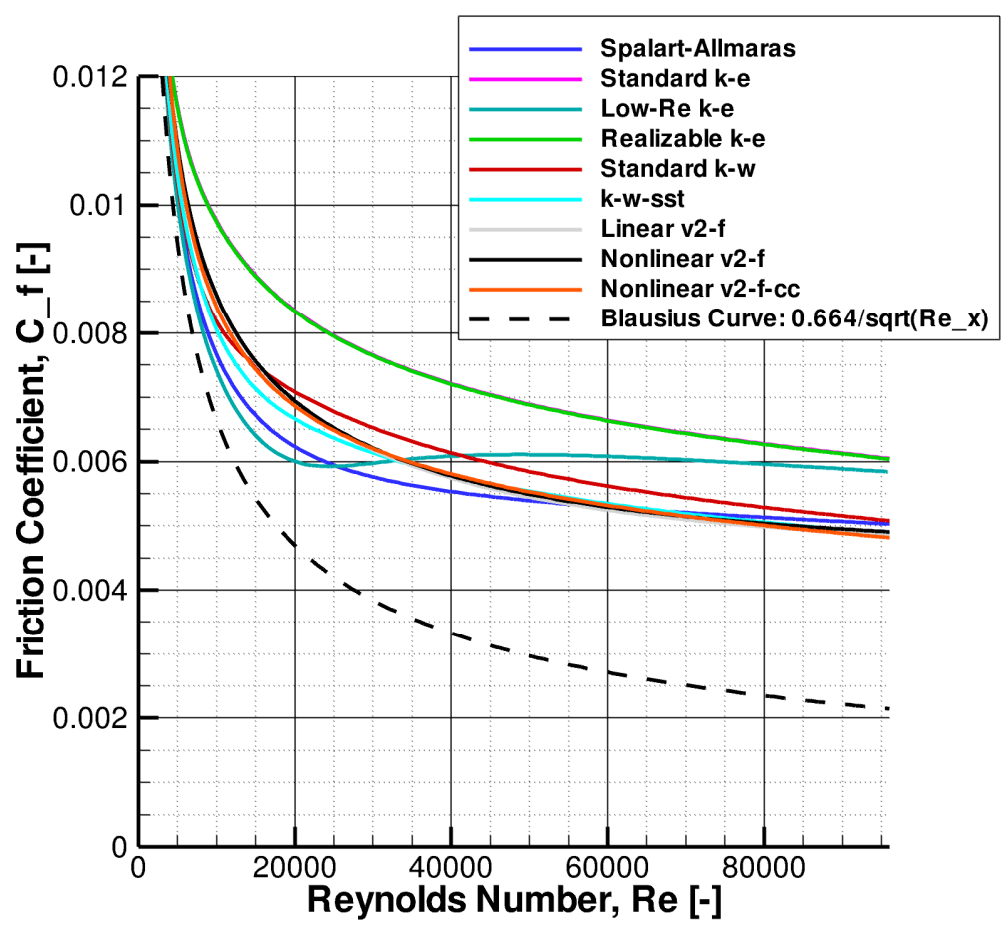

Figure 2. Skin friction coefficient distribution for a laminar flat plate in subsonic flow.

The $v^{2}-f$ turbulence models all predict essentially identical skin friction coefficient distributions, indicating that the nonlinear models reduce to the standard linear model in simple flows. The $v^{2}-f$ models show similar near-wall flow characteristics to standard models, including skin friction coefficient, indicating that the models are capturing similar effects as other well-documented and validated turbulence models.

\section{B. Validation Case: Flat Plate in Turbulent, Subsonic Flow}

The three $v^{2}-f$ models and several common turbulence models were again used to solve a subsonic flow over the same flat plate, but the fluid viscosity was reduced to quicken the transition to turbulent flow. The Reynolds number at the end of the plate is $1 \times 10^{7}$, indicating turbulent flow (transition to turbulence occurs at $5 \%$ of the chord.) The skin friction coefficient generated using the $v^{2}-f$ turbulence models, several common turbulence models, and a theoretical relationship are shown in the following figure. The theoretical relationship is Prandtl's well-known relationship for turbulent flat plate skin friction coefficient ${ }^{40}$,

$$
C_{f}=\frac{0.027}{R e_{x}^{1 / 7}}
$$

$10-$

American Institute of Aeronautics and Astronautics -

2010-1054 


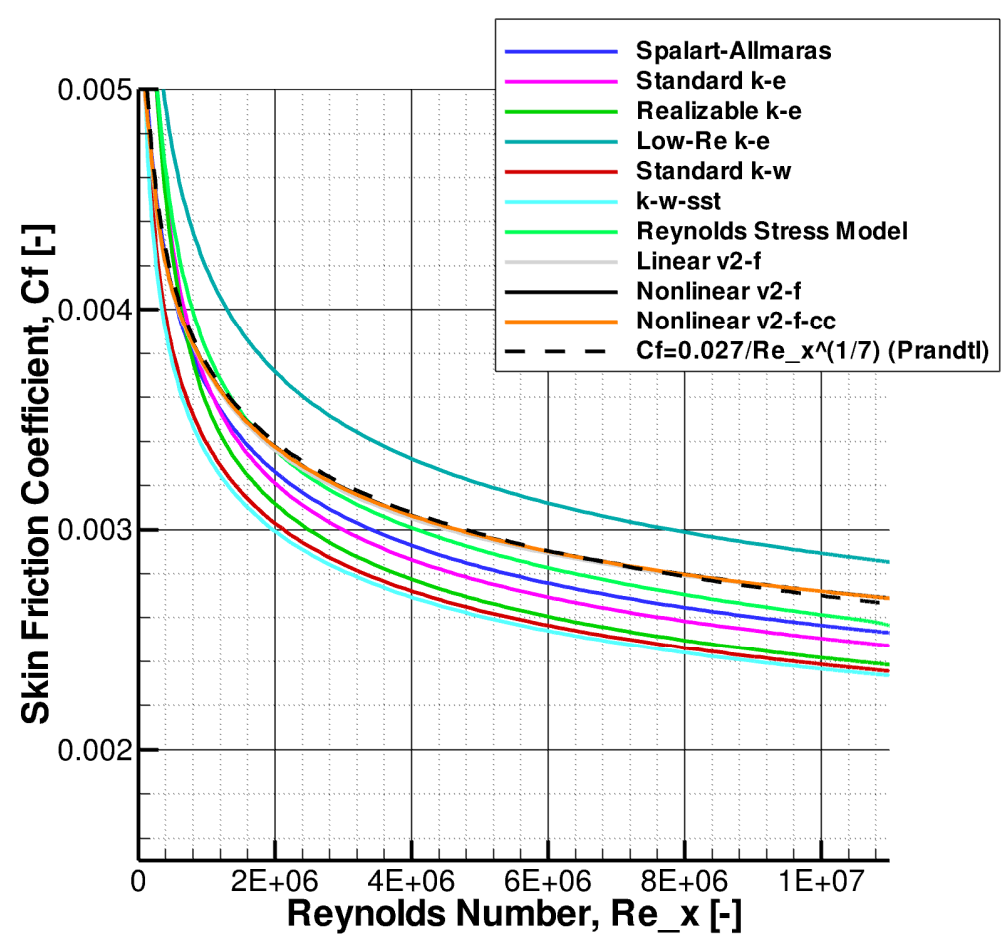

Figure 3. Skin friction coefficient distribution for a turbulent flat plate in subsonic flow.

The three $v^{2}-f$ turbulence models predict nearly identical skin friction coefficient distributions, again indicating that the models reduce to the base model in simple flows. Further, the models match Prandtl's skin friction coefficient distribution nearly exactly.

\section{General Aviation Circulation Control Airfoil}

As has been repeatedly demonstrated, common turbulence models are ill-suited for circulation control flows. This can be due to inappropriate simplification of flows (in the case of zero-, one-, or two-equation eddy viscosity models) or due to tight coupling of Reynolds stress transport equations (in the case of Reynolds stress transport models.) In general, CFD solutions using standard eddy viscosity models showed a significant over-prediction of integrated quantities, including the lift coefficient. For example, the following figures show results from Jones et al. ${ }^{42}$, Lee-Rausch et al. ${ }^{22}$, and Swanson et al. ${ }^{24}$ 

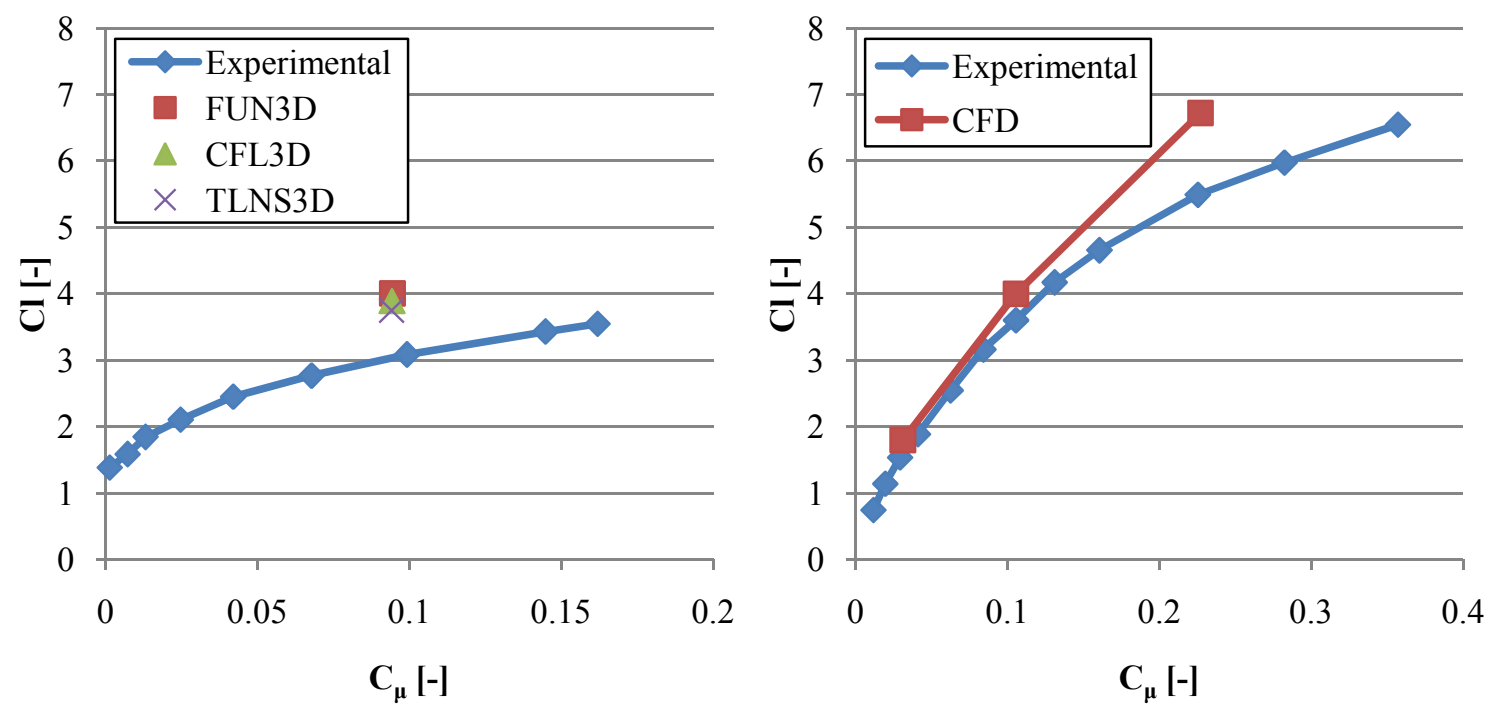

Figure 4. Circulation control results from Lee-Rausch et al. (left) and Jones et al. (right)

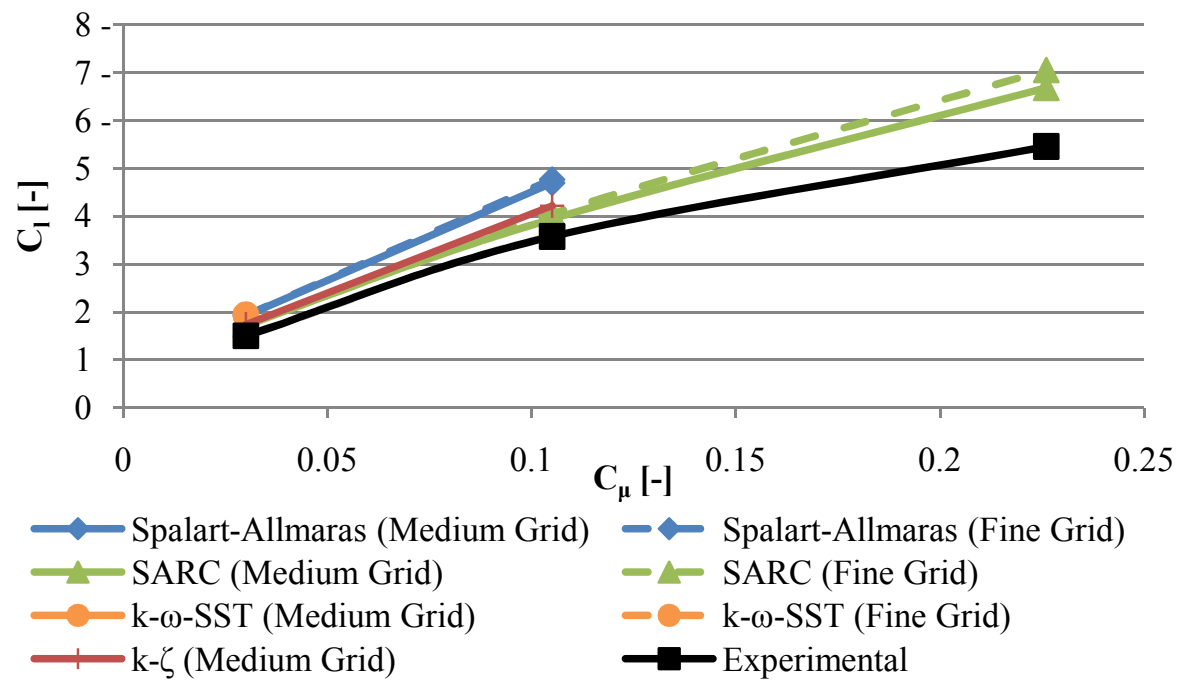

Figure 5. Circulation control results from Swanson et al.

The $v^{2}-f$ turbulence models were applied to the General Aviation Circulation Control airfoil (the same airfoil used in the studies by Jones et al. and Lee-Rausch et al.) Because of the increased robustness of the standard $v^{2}-f$ model, it was expected that these models should yield increased accuracy in complex circulation control flows. Further, results were generated using more common turbulence models to verify the results presented by LeeRausch et al. and Jones et al.

Careful attention was paid to the grid generation process to limit errors due to poor gridding. In particular, several aspects of the grid were scrutinized. First, gridline orthogonality needed to be enforced to minimize numerical error. This criteria, along with the large flap deflection, led to a unique farfield configuration; this was necessary to provide adequate mapping of gridlines from both the flap and the near-flap region on the lower surface to the farfield. Second, since the $v^{2}-f$ model does not use damping functions nor wall functions, the cell nearest any wall needed to be placed in the laminar sublayer (generally $y^{+}<5$.) Third, the leading edge discretization needed to be sufficient to capture the stagnation point, which is crucial in predicting the lift coefficient. Finally, the grid needed sufficient resolution in the wake region to capture recirculation, should the $v^{2}-f$ turbulence model predict it. A fully structured grid was generated to meet these criteria. The computational grid is shown in the following figures.

12 -

American Institute of Aeronautics and Astronautics 2010-1054 




13 -

American Institute of Aeronautics and Astronautics 2010-1054 
Figure 6. Computational grid for GACC airfoil: farfield (top-left); nearfield (top-right); leading edge (middle-left); flap (middle-right); circulation control slot (bottom.)

The $v^{2}-f$ turbulence models show some improvement in the prediction of the lift coefficient for circulation control airfoils, and the results obtained using common turbulence models showed the same over-prediction that Jones et al. and Lee-Rausch et al. showed. Preliminary results are shown below.
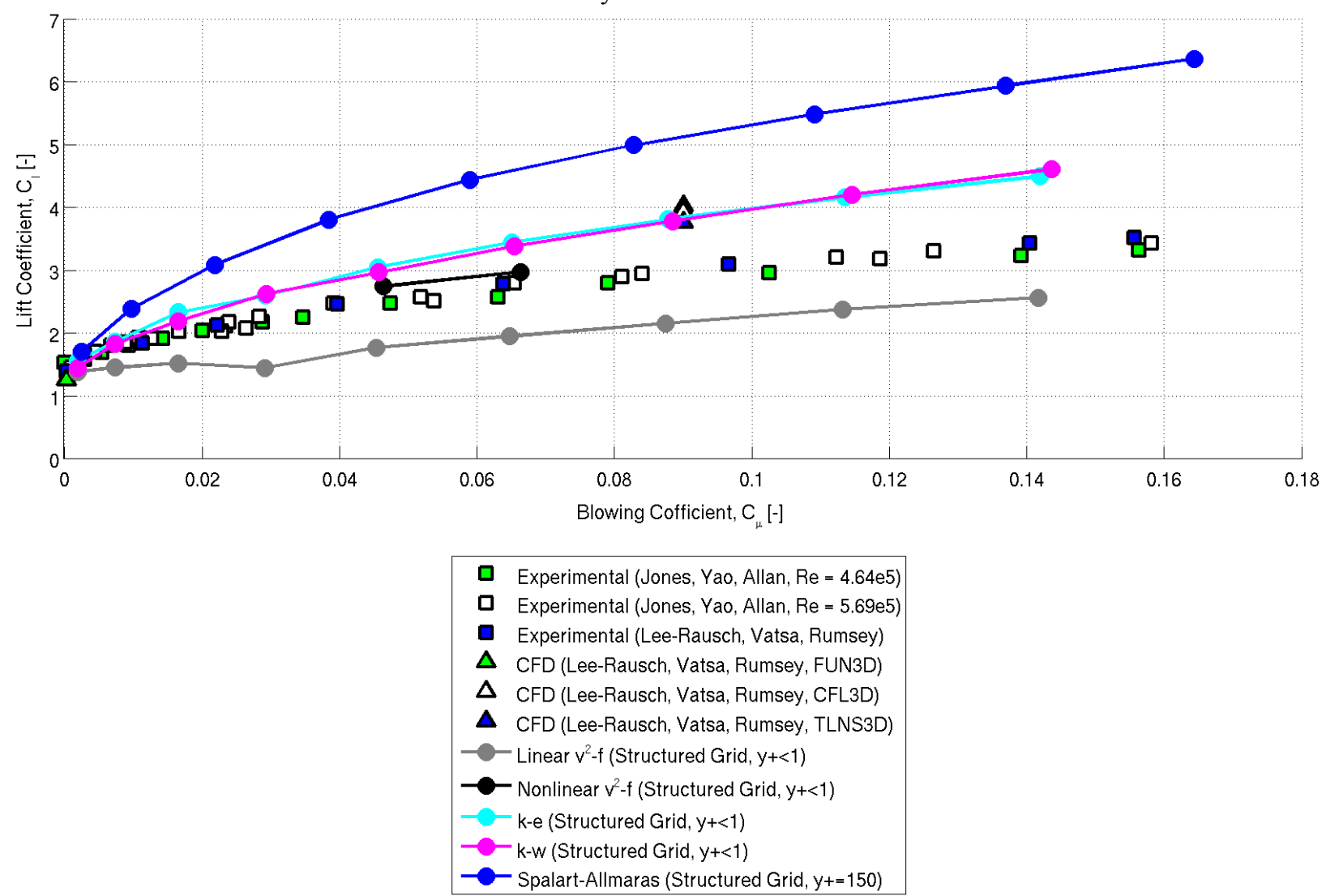

Figure 7. CFD results for the $v^{2}$ - $f$ turbulence model compared to common turbulence models and experimental data

\section{Conclusions and Future Work}

The linear $v^{2}$-f turbulence model yields results that are only marginally more accurate than those produced by standard turbulence models. Clearly, the addition of the velocity scale and elliptic relaxation terms lowers the integrated coefficients, though it lowers them too much. The physical source of this is unknown as of yet, and should be researched to further understanding of both circulation control flows and the $v^{2}-f$ turbulence model. It is important to note, though, that the solutions for the linear $v^{2}-f$ model shown in Figure 7 are not entirely converged, and the lift coefficient history shows an upward trend, especially in the high blowing coefficient regime, as is shown in Figure 8. Iterating these cases further will likely improve the results.

14 -

American Institute of Aeronautics and Astronautics 2010-1054 


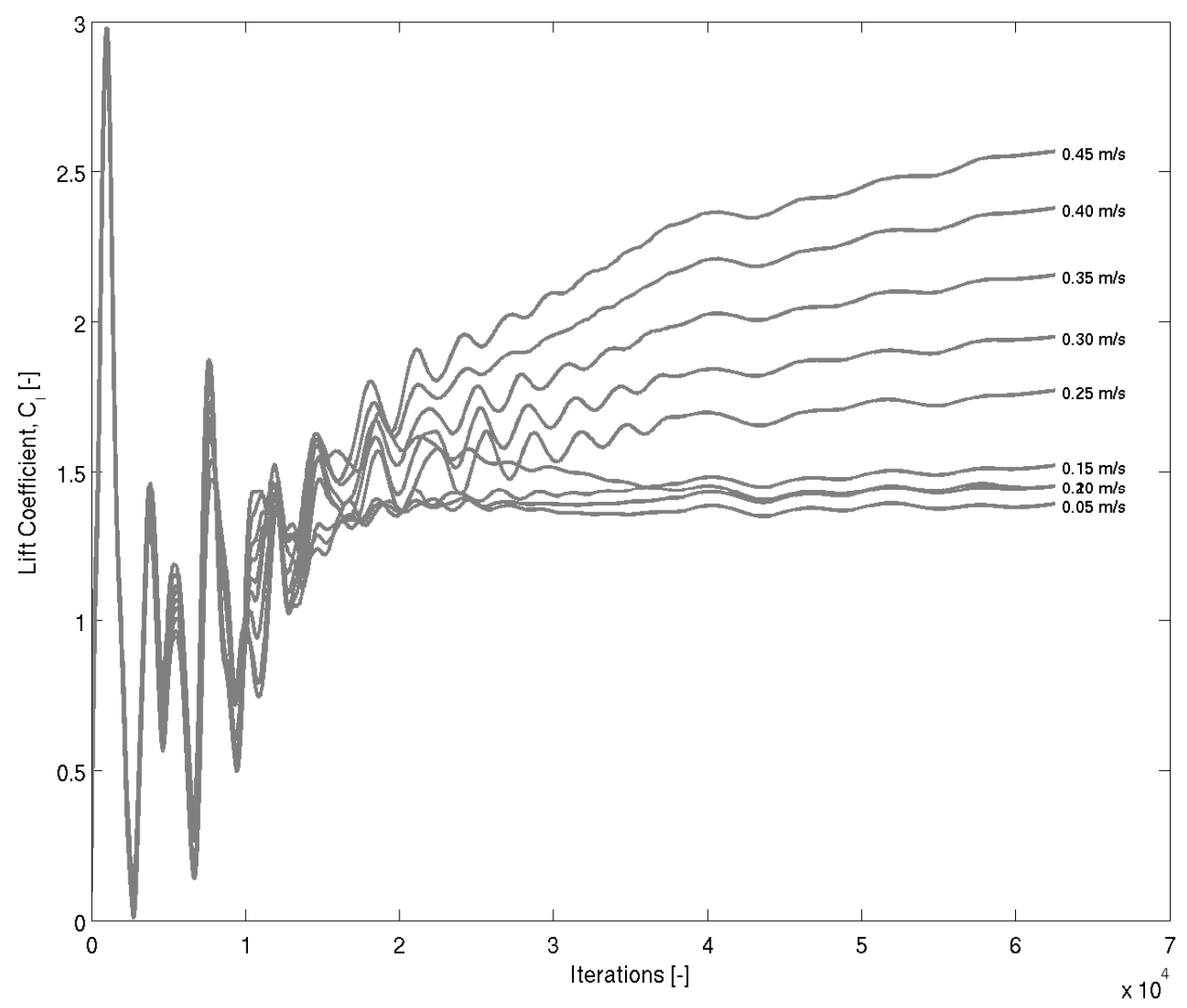

Figure 8. Lift coefficient history for the linear $v^{2}-f$ model

The nonlinear $v^{2}-f$ turbulence model improves modeling of the GACC airfoil. The nonlinearization of the turbulent viscosity, in addition to the inclusion of the velocity scale and the elliptic relaxation factor, appear to yield improved results for the lift coefficient. However, more solutions need to be developed to ensure that this claim is valid over a range of blowing coefficients. The effect of the nonlinearization of the turbulent viscosity without the inclusion of the velocity scale and elliptic relaxation terms (i.e., the nonlinearization of the $k-\varepsilon$ turbulence model,) would be an interesting and enlightening task.

Applying the $v^{2}-f$ turbulence model with a nonlinear turbulent viscosity formulation and streamline curvature correction shows theoretical potential to further improve predictions, though this claim needs to be validated. In the near future, this version of the $v^{2}-f$ turbulence model will be included in Figure 7.

\section{Acknowledgements}

This work was funded as part of a NASA Research Announcement award under Contract \#NNL07AA55C with Craig Hange and Clif Horne as the technical monitors. Special thanks to Dr. Bjorn Anders Pettersson-Reif, Dr. Karthik Duraisamy, Greg Jones, Elizabeth Lee-Rausch, and Christian Heschl for their respective contributions to this work.

\section{References}

1. Englar, R. J., and Huson, G. G., "Development of Advanced Circulation Control Wing High Lift Airfoils," AIAA 831847, 1983.

2. Panitz, T., and Wasan, D. T., "Flow Attachment to Solid Surfaces: the Coanda Effect," AIChE Journal vol. 18 no. 1 , 1972 .

15 -

American Institute of Aeronautics and Astronautics 2010-1054 
3. Golden, R., "Design and Performance of Circulation Control Dual Radius Flap Systems," Aerospace Engineering Department, California Polytechnic State University (unpublished).

4. Wallin, S., and Johansson, A. V., "Modelling Streamline Curvature Effects in Explicit Algebraic Reynolds Stress Turbulence Models," International Journal of Heat and Fluid Flow vol. 23, 2002.

5. Spalart, P. R., and Shur, M., "On the Sensitization of Turbulence Models to Rotation and Curvature," Aerospace Science and Technology vol. 4, 1997.

6. Gatski, T., and Jongen, T., "Nonlinear Eddy Viscosity and Algebraic Stress Models for Solving Complex Turbulent Flows," Prog. Aero. Sci. vol. 36, 2000.

7. Hellsten, A., Wallin, S., and Laine, S., "Scrutinizing Curvature Corrections for Algebraic Reynolds Stress Models," AIAA Paper 2002-2963, 2002.

8. Craft, T. J., "Nonlinear Eddy-Viscosity Models," Department of Mechanical, Aerospace, and Manufacturing Engineering, University of Manchester, Manchester, England (unpublished).

9. Smith, A. M. O., and Cebeci, T., "Numerical Solution of the Turbulent Boundary Layer Equations," Douglas Aircraft Division Report DAC 33735, 1967.

10. Baldwin, B. S., and Lomax, H., "Thin Layer Approximation and Algebraic Model for Separated Turbulent Flows," AIAA Paper 78-257, 1978.

11. Spalart, P. R., and Allmaras, S. R., “A One-Equation Turbulence Model for Aerodynamic Flows,” AIAA Paper 92-0439, 1992.

12. Harlow, F. H., and Nakayama, P. I., "Transport of Turbulence Energy Decay Rate," Los Alamos Sci. Lab., LA-3854, 1968.

13. Wilcox, D. C., "Reassessment of the Scale-Determining Equation for Advanced Turbulence Models," AIAA Journal vol. 26, pp. 1299-1310, 1988.

14. Durbin, P. A., "Near-Wall Turbulence Closure without 'Damping Functions'," Theoretical and Computational Fluid Dynamics 3:1-13, 1991.

15. Pope, S. B., “A More General Eddy-Viscosity Hypothesis,” Journal of Fluid Mechanics vol. 72, 1975.

16. Launder, B. E., and Sandham, N. D., Closure Strategies for Turbulent and Transitional Flows, Cambridge University Press, New York, 2002.

17. Jingchang, L., Mao, S., and Liyi, W., "Navier-Stokes Analysis of a Circulation Control Airfoil," ACTA Mechanica Sinica, vol. 11, no. 2, 1995.

18. Liu, Y., "Numerical Simulations of the Aerodynamic Characteristics of Circulation Control Wing Sections," Department of Aerospace Engineering, Georgia Institute of Technology, 2003.

19. Liu, Y., Sankar, L. N., Englar, R. J., and Ahuja, K. K., "Numerical Simulations of the Steady and Unsteady Characteristics of a Circulation Control Wing Airfoil,” AIAA Paper 2001-0704, 2001.

20. McGowan, G., and Gopalarathnam, A., "CFD Analysis of a Circulation Control Airfoil Using Fluent," Mechanical and Aerospace Engineering Department, North Carolina State University, 2004.

21. Ball, T., "CFD as Applied to the Design of Short Takeoff and Landing Vehicles Using Circulation Control," Aerospace Engineering Department, California Polytechnic State University, 2008.

22. Lee-Rausch, E. M., Vatsa, V. N., and Rumsey, C. L., "Computational Analysis of Dual Radius Circulation Control Airfoils," AIAA Paper 2006-3012, 2006.

23. Pfingsten, K. C., Jensch, C., Korber, K. W., and Radespiel, R., "Numerical Simulation of the Flow around Circulation Control Airfoils," First CEAS European Air and Space Conference, 2007.

24. Swanson, R. C., Rumsey, C. L., Anders, S. G, "Progress Towards Computational Method for Circulation Control Airfoils," AIAA Paper 2005-29, 2005.

25. Chang, P. A., Slomski, J., Marino, T., and Ebert, M. P., "Numerical Simulation of Two- and Three-Dimensional Circulation Control Problems," $43^{\text {rd }}$ AIAA Aerospace Sciences Meeting and Exhibit, 2005.

26. McGowan, G., Gopalarathnam, A., and Jones, S., "Analytical and Computational Study of Adaptive Circulation Control Airfoils," AIAA 2004-4721, 2004.

27. Durbin, P. “Application of a Near-wall Turbulence Model to Boundary Layers and Heat Transfer," International Journal of Heat and Fluid Flow vol. 14, 1993.

28. Bell, Br., "Turbulent Flow Case Studies," Fluent Services Center, 2003.

29. Parneix, S., Durbin, P. A, and Behnia, M., "Computation of 3-D Turbulent Boundary Layers using the V2F Model," Flow, Turbulence, and Combustion vol. 60, 1998.

30. Behnia, M., Parneix, S., and Durbin, P., "Prediction of Heat Transfer in a Jet Impinging on a Flat Plate," International Journal of Heat and Mass Transfer vol. 41, 1998.

31. Kalitzin, G., "Application of the $v^{2}-f$ Model to Aerospace Configurations," Center for Turbulence Research Annual Research Briefs, 1999.

32. Pettersson Reif, B. A., "A Nonlinear Constitutive Relationship for the $v^{2}-f$ Model," center for Turbulence Research Annual Research Briefs, 1999.

33. Wilcox, D. C., Turbulence Modeling for CFD, 3rd ed., DCW Industries, California, 2006.

34. Pettersson Reif, B. A., Durbin, P. A., and Ooi, A., "Modeling Rotational Effects in Eddy-Viscosity Closures," International Journal of Heat and Fluid Flow, 1999.

16 -

American Institute of Aeronautics and Astronautics 2010-1054 
35. Heschl, Ch., Sanz, W., Klanatsky, P1, and Madou, F., "Comparison of Different Turbulence Models to Compute Wall Affected Room Airflows," TU Graz, Institute for Turbomachinery and Machine Dynamics, 2005.

36. Hellsten, A., Wallin, S., and Laine, S., "Scrutinizing Curvature Corrections for Algebraic Reynolds Stress Models," AIAA Paper 2002-2963, 2002.

37. Chow, J. S., Zilliac, G. G., and Bradshaw, P., "Mean and Turbulence Measurements in the Near Field of a Wingtip Vortex," AIAA Journal 35, 1997.

38. ANSYS ${ }^{\circ}$ FLUENT, v. 6.3.26.

39. Heschl, Ch., Sanz, W., and Klanatsky, P., "Implementation and Comparison of Different Turbulence Models for Three Dimensional Wall Jets with FLUENT," TU Graz, Institute for Thermal Turbomachinery and Machine Dynamics, 2005.

40. Kundu, P. K., Fluid Mechanics, Academic Press, London, 1990.

41. Duraisamy, K., and Iaccarino, G., "Curvature Correction and Application of the $v^{2}-f$ Turbulence Model to tip Vortex Flows," Center for Turbulence Research Annual Research Briefs, 2005.

42. Jones, G. S., Lin, J. C., Allan, B. G., Milholen, W. E., Rumsey, C. L., and Swanson, R. C., "Overview of CFD Validation Experiments for Circulation Control Applications at NASA," NASA 20080031119, 2008.

43. White, F. M., Viscous Fluid Flow, McGraw-Hill, New York, 2006.

44. Storm, T., "Assessing the $v^{2}-f$ Turbulence Models for Circulation Control Applications," Aerospace Engineering Department, California Polytechnic State University (unpublished). 